\title{
Fiber Loop Mirror Sensors Interrogated and Multiplexed by OTDR
}

\author{
Maria Thereza Miranda Rocco Giraldi, Cindy Stella Fernandes, Marta S. Ferreira, Student Member, OSA, \\ Marco José de Sousa, Pedro A. S. Jorge, João Crisóstomo Weyl Albuquerque Costa, \\ José Luís Campos Oliveira Santos, Member, OSA, and Orlando Frazão, Senior Member, OSA
}

\begin{abstract}
In this paper, two techniques for interrogation and multiplexing of fiber loop mirror (FLM) intensity sensors based on optical time domain reflectometer (OTDR) are proposed. These configurations enable series and parallel FLM sensor interrogation. A fiber taper characterized as a displacement sensor was used as the intensity sensor. The OTDR parameters were optimized in order to obtain the best results. The optimized parameters were 100-ns pulse width and 10-dB input signal attenuation which permitted to attain $\sim 18 \mathrm{~dB}$ dynamic range in the operating wavelength of $1550 \mathrm{~nm}$. The results show a linear behavior for both configurations with similar slope, $-15.3 \mathrm{~dB} / \mathrm{mm}$, in the normalized displacement range of 0.2 to $0.7 \mathrm{~mm}$. It was also achieved a displacement resolution of 0.027 and $0.093 \mathrm{~mm}$, for the series and parallel configurations, respectively. Sensors multiplexing are demonstrated for both configurations and the systems do not present crosstalk. Based on the experimental results, the best configuration is the parallel one. The proposed approach is a viable alternative for multiplexing and interrogation of remote fiber sensors.
\end{abstract}

Index Terms-Fiber loop mirror (FLM), optical fiber sensor, OTDR, sensor interrogation.

\section{INTRODUCTION}

O PTICAL time domain reflectometer (OTDR) is the most utilized equipment to measure distributed losses in optical fibers. It uses Rayleigh scattered light to determine the attenuation of optical fiber links. OTDR is also useful to localize events, breaks and to evaluate splices and connectors [1].

Manuscript received July 23, 2014; revised September 9, 2014; accepted September 22, 2014. Date of publication September 25, 2014; date of current version April 29, 2015. This work was supported in part by CAPES Project BEX 4463/13-7, Conselho Nacional de Desenvolvimento Científico e Tecnológico, European Regional Development Fund through the COMPETE Program (Operational Program for Competitiveness), and by National Funds through the FCT-Fundação para a Ciência e Tecnologia (Portuguese Foundation for Science and Technology) within Project FCOMP-01-0124-FEDER-037281.

M. T. M. R. Giraldi is with the Institute for Systems and Computer Engineering of Porto, Porto 4169-007, Portugal, on leave from the Military Institute of Engineering, Rio de Janeiro 22.290-270, Brazil (e-mail: mtmrocco@ime.eb.br)

C. S. Fernandes is with the Institute for Systems and Computer Engineering of Porto, Porto 4169-007, Portugal, on leave from the Federal University of Pará, Belém 66.075-970, Brazil (e-mail: cindy.fernandes@ gmail.com).

M. S. Ferreira and P. A. S. Jorge are with the Institute for Systems and Computer Engineering of Porto, Porto 4169-007, Portugal (e-mail: msferreira@ inescporto.pt; pedro.jorge@fc.up.pt).

M. J. de Sousa and J. C. W. A. Costa are with the Federal University of Pará, Belem 66.075-970, Brazil (e-mail: marcojsousa@gmail.com; joao. weyl@gmail.com).

J. L. C. O. Santos and O. Frazão are with the Institute for Systems and Computer Engineering of Porto, Porto 4169-007, Portugal, and also with the Faculty of Sciences of University of Porto, Porto 4099-002, Portugal (e-mail: josantos@ fc.up.pt; ofrazao@fc.up.pt).

Color versions of one or more of the figures in this paper are available online at http://ieeexplore.iee.org.

Digital Object Identifier 10.1109/JLT.2014.2360500
Due to these advantages and since OTDR is a simple, easy and ready to be used tool, it has also been the starting point of distribution sensing techniques [1]. As interrogation system, OTDR has been utilized in different configurations. One of the most common is to use fiber Bragg grating (FBG) and/or long period grating (LPG) with OTDR [2]-[4]. Recently, it was proposed a multi-point strain measurement system based on OTDR for FBG sensors [2], [3]. Another approach employs OTDR to interrogate Fabry-Perot cavities sensors [5], [6]. Finally, a significant function of the OTDR is to enable multiplexing [7]-[9] and remote sensing [8]-[10] interrogation. OTDR trace loss [8], [11]-[13] or reflection peak variation [7], [10] are the most attractive techniques for interrogation when OTDR is used. In the case of the OTDR trace loss and considering multiplexing and remote sensing, there is a compromise between the number of sensors to be multiplexed and the distance between sensors since the loss introduced by the sensors will be a serious limitation to the system. OTDR reflection peak variation does not present such limitation.

An optical fiber mirror is a device necessary in several applications. The most important application is to be used as sensing element. One of the solutions is to utilize a fiber loop mirror (FLM) which is straight forward developed using a $3 \mathrm{~dB}$ optical coupler. In this case, the output ports of the coupler are spliced together forming a loop. All light input to the loop is reflected back to the coupler input port due to constructive interference which occurs in the coupler after the counter waves propagate inside the loop [14]. The optical losses are merely a result of the $3 \mathrm{~dB}$ coupler insertion loss and the splice loss. Fiber ring combined with LPG is an alternative solution to interrogate such structure in reflection using an OTDR as the interrogation system [15]. The FLM sensor could operate both in transmission and reflection, and its functioning does not depend on the light polarization at the input port. When compared with other temperature sensors, this configuration presented higher sensitivity [16], [17]. Strain sensor based on a Hi-Bi FLM was also investigated [18]. In 2004, temperature dependence of a polarization maintaining (PM) photonic crystal fiber in a FLM was also demonstrated. Comparing with a standard PM fiber, reduced temperature sensitivity was obtained [19]. In 2005, the characteristics of a Hi-Bi FLM composed of a standard $3 \mathrm{~dB}$ fiber coupler with one or more sections of Hi-Bi fibers were discussed. Displacement and temperature sensors were theoretically and experimentally analyzed. Using the characteristics of the $\mathrm{Hi}-\mathrm{Bi}$ FLM, a strain-temperature sensor was proposed and demonstrated as an interrogation system for new applications [20]. 


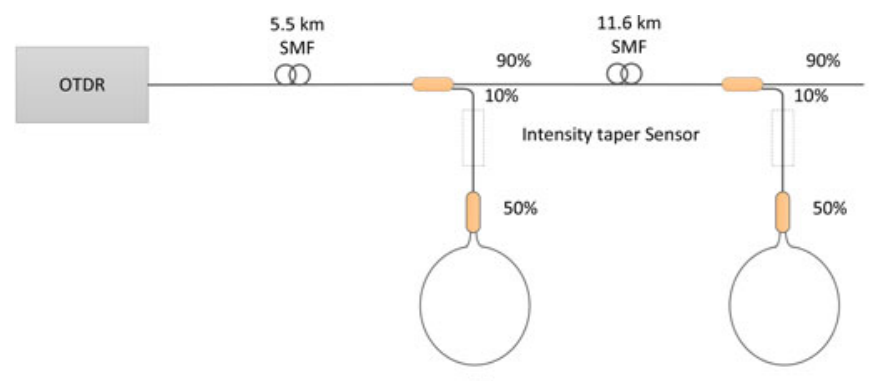

(a)

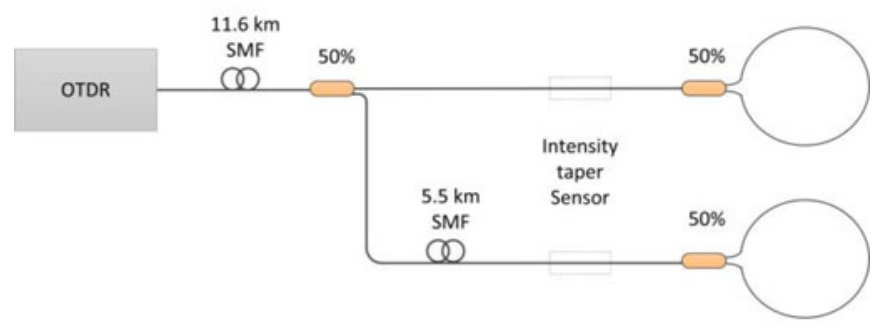

(b)

Fig. 1. Experimental setups of the (a) series and (b) parallel interrogation and multiplexing systems for FLM intensity sensors using OTDR.

In this paper, two interrogation and multiplexing systems based on OTDR are presented to address FLM intensity sensors. The FLMs are interrogated in series or in parallel when located along the fiber line. A peak reflection is generated due to the Rayleigh scattering combined with the light reflected by the FLM. In this case, when the losses are generated by the sensors the light reflected by the peak reflection of the FLM decreases with the applied physical parameter. A fiber taper is used as displacement sensor and is located before the FLMs.

\section{EXPERIMENTAL SETUP}

The proposed interrogation techniques based on OTDR enable to multiplex several intensity sensors along the fiber. This feature is illustrated in Fig. 1(a) for the series configuration, which shows a system with two FLM intensity sensors. Considering the $2.5 \mathrm{~dB}$ loss originated by the $90 \times 10$ optical coupler insertion loss, it is possible to attain multiplexing of around ten sensors. For the parallel configuration, multiplexing is achieved by using a splitter which will divide the optical signal in as much sensors as needed, depending on the number of output ports of the splitter and the signal level. One interesting solution to solve this drawback is to use an optical switch instead of the splitter. In this case, the number of sensors will be dependent merely on the interrogation time. In Fig. 1(b), the splitter is a $1 \times 2$ device and therefore it is shown two multiplexed FLM intensity sensors. Note that the length of the fibers after the splitter must be different in order to allow sensors discrimination. Another interesting possibility of such proposed systems is that they allow using both configurations together in a hybrid multiplexing scheme.

The experimental setups used to build the two proposed interrogation systems are presented in Fig. 2. A commercial OTDR from YOKOGAWA, Inc model AQ 1200 OTDR-Multi Field Tester is used to interrogate intensity taper sensors. Remote

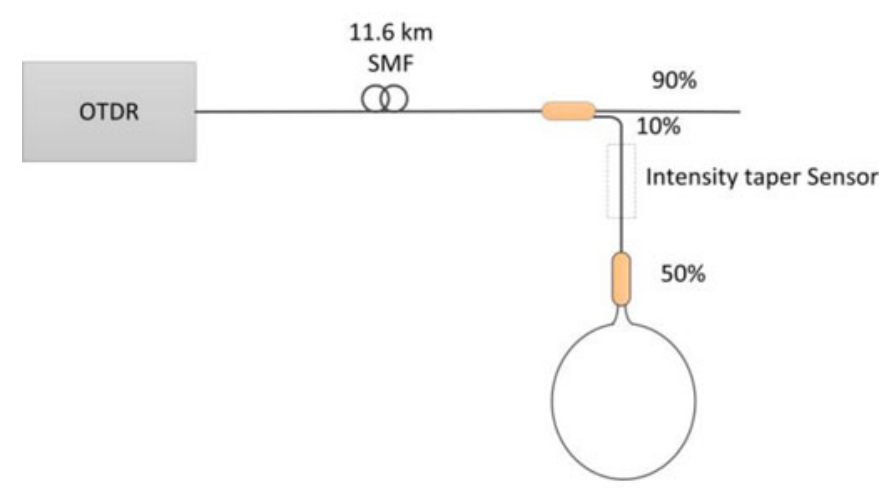

(a)

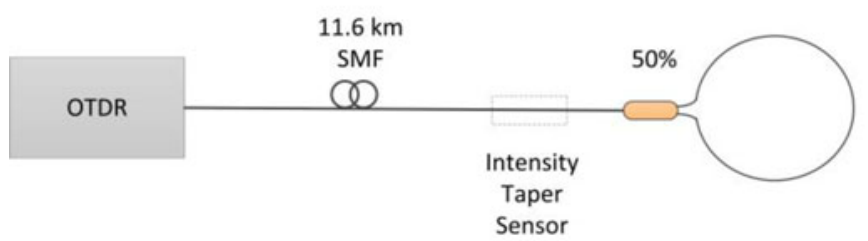

(b)

Fig. 2. Experimental setups used to build the two proposed interrogation systems when (a) series and (b) parallel multiplexing interrogation systems are concerned.

sensing is obtained connecting $11.6 \mathrm{~km}$ of Corning SMF-28 fiber to the OTDR port. In Fig. 2(a), an optical coupler is connected at the fiber output. Ten percent optical power is used to illuminate the sensor. A $3 \mathrm{~dB}$ optical coupler working as a FLM is connected to the output of the displacement sensor. The $90 \%$ output port of the coupler enables multiplexing. This configuration permits a series sensor interrogation, when sensors multiplexing are concerned. Fig. 2(b) shows the other interrogation system proposed, where the intensity sensor is connected to the end of the $11.6 \mathrm{~km}$ Corning SMF-28 fiber directly. The FLM is also attached to the output of the displacement sensor. This configuration enables a parallel sensor interrogation, when sensors multiplexing are involved. To simulate the intensity sensor, an optical fiber taper was used as the displacement sensor. The operation mode of the sensing head consists in applying curvature in the taper region with a value that depends on the displacement to be measured. Therefore, the displacement induced curvature variation originates a change in the insertion loss of the taper. At first, both configurations are similar, but when sensors multiplexing are concerned, they behave differently.

\section{OPTIMIZATION SigNALS}

In order to optimize the OTDR parameters (optical signal) it was evaluated the dynamic range of the FLM reflection peak by varying the input signal and its pulse width. The FLM reflection peak dynamic range as a function of the input signal attenuation is shown in Fig. 3. It is observed that an input signal attenuation of $10 \mathrm{~dB}$ allows obtaining a maximum dynamic range of around $17 \mathrm{~dB}$. The peak signal is not saturated due to the optical coupler $90 \times 10$ located before the FLM. Fig. 4 illustrates the FLM reflection peak dynamic range as a function of the OTDR 


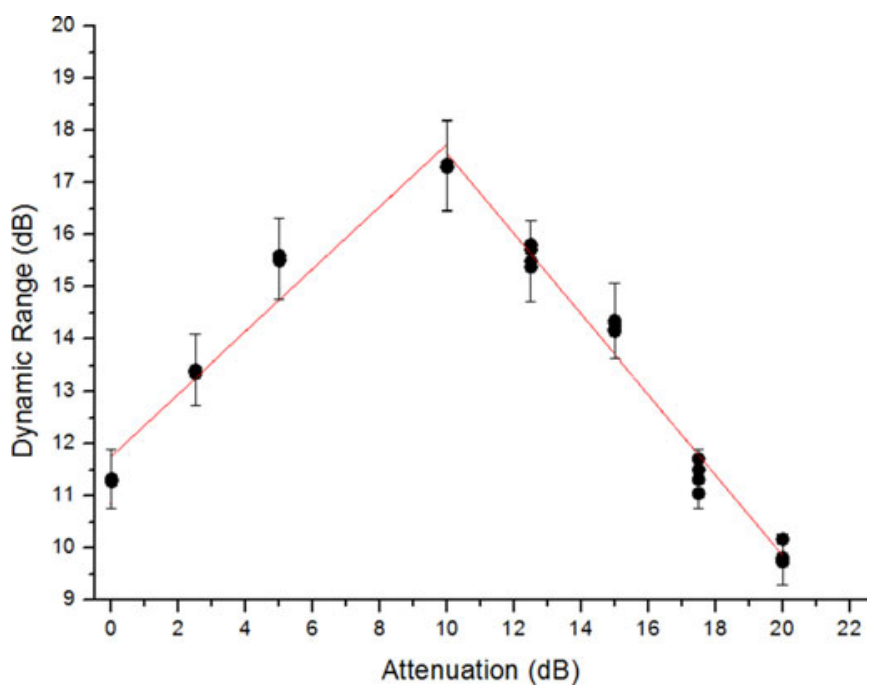

Fig. 3. Dynamic range of the FLM reflection peak as a function of the input signal attenuation.

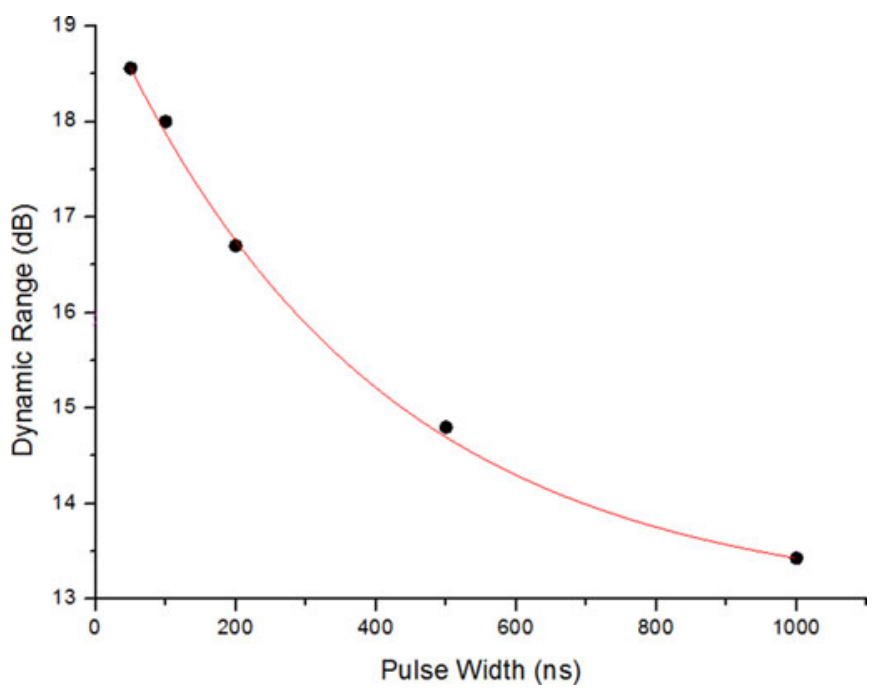

Fig. 4. Dynamic range of the FLM reflection peak as a function of pulse width.

input signal pulse width. It is verified that smaller pulse width results in higher dynamic range. A pulse width of $\sim 100 \mathrm{~ns}$ enabled to attain an even better dynamic range of $\sim 18 \mathrm{~dB}$. In both curves, the dots represent the experimental results and the solid lines are the fitting curves. It was compared the behavior of both interrogation systems with the OTDR operating at 1550 and $1300 \mathrm{~nm}$. Since the attenuation is lower at $1550 \mathrm{~nm}$, this wavelength presented better results and was therefore used throughout this work.

The OTDR traces for the two interrogation systems configurations can be observed in Fig. 5. It is seen a $2.5 \mathrm{~dB}$ difference between the OTDR reflection peak curves, as better observed in the inset. Such difference is due to the $90 \times 10$ optical coupler used in the series configuration [see Fig. 2(a)] which enables only $10 \%$ of the signal to be input to the sensor and after that to be reflected by the FLM. On the other hand, the parallel config-

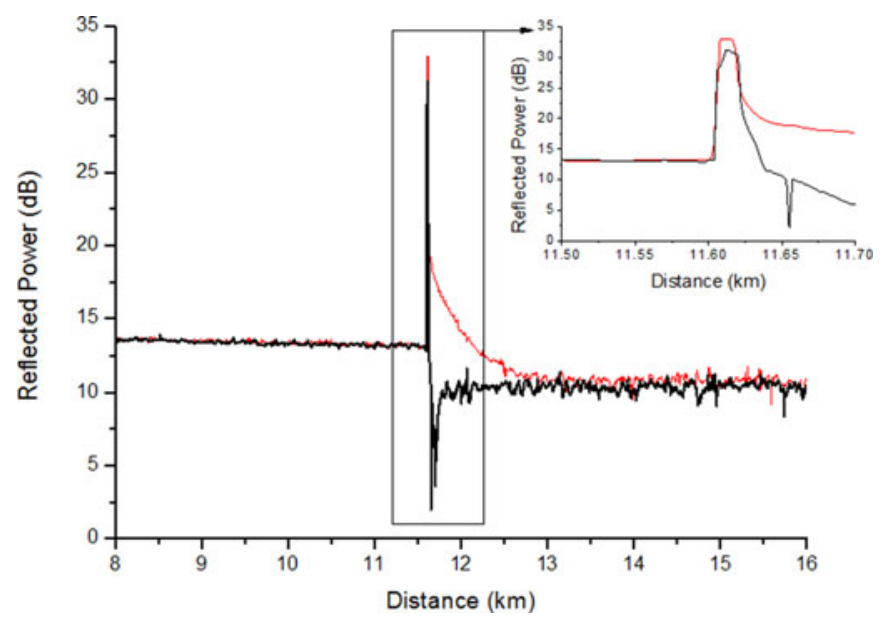

Fig. 5. OTDR traces for the parallel (line) and series (bold line) interrogation systems. Inset: better view of the OTDR peak curves.

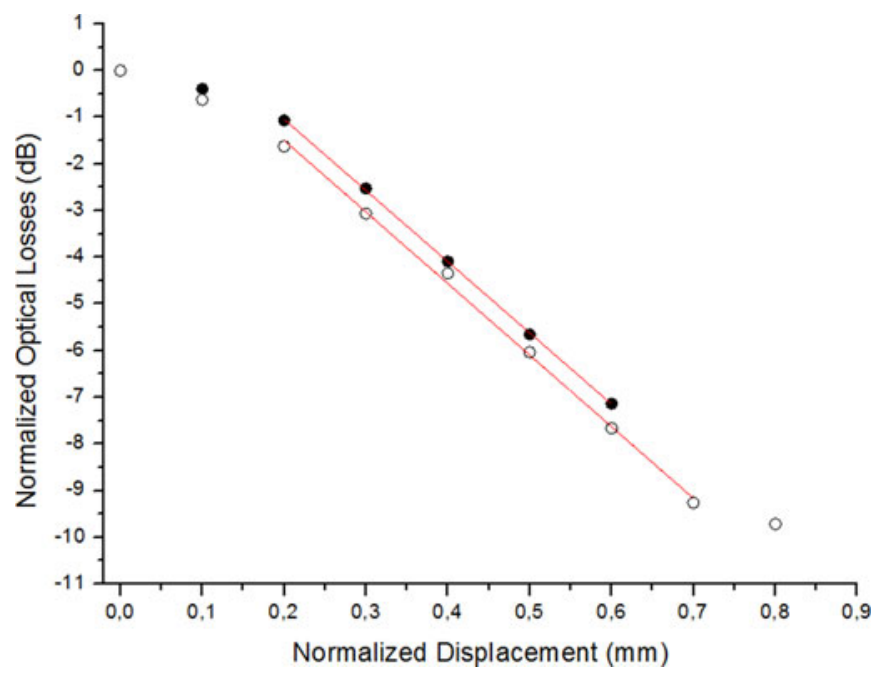

Fig. 6. Normalized optical losses in the FLM reflection peak as a function of the normalized displacement for the parallel (solid circles) and series (open circles) configurations.

uration [see Fig. 2(b)] enables all signals to input the sensor and to be reflected. However, since this configuration reflects the entire signal, such reflected signal is intense and suffers further reflection back and forth.

\section{EXPERIMENTAL RESULTS AND DISCUSSION}

The normalized optical losses in the FLM reflection peak as a function of the normalized displacement sensor for the parallel (solid circles) and series (open circles) configurations are presented in Fig. 6. The results evidence similar linear behavior within the normalized displacement range from 0.2 to $0.7 \mathrm{~mm}$ with a slope of around $-15.3 \mathrm{~dB} / \mathrm{mm}$.

The sensing heads resolutions with the proposed interrogation systems were obtained by performing a step of 0.4 (series configuration) and $0.3 \mathrm{~mm}$ (parallel configuration) in the displacement sensor which corresponds to a 3.91 and $4.19 \mathrm{~dB}$ signal 


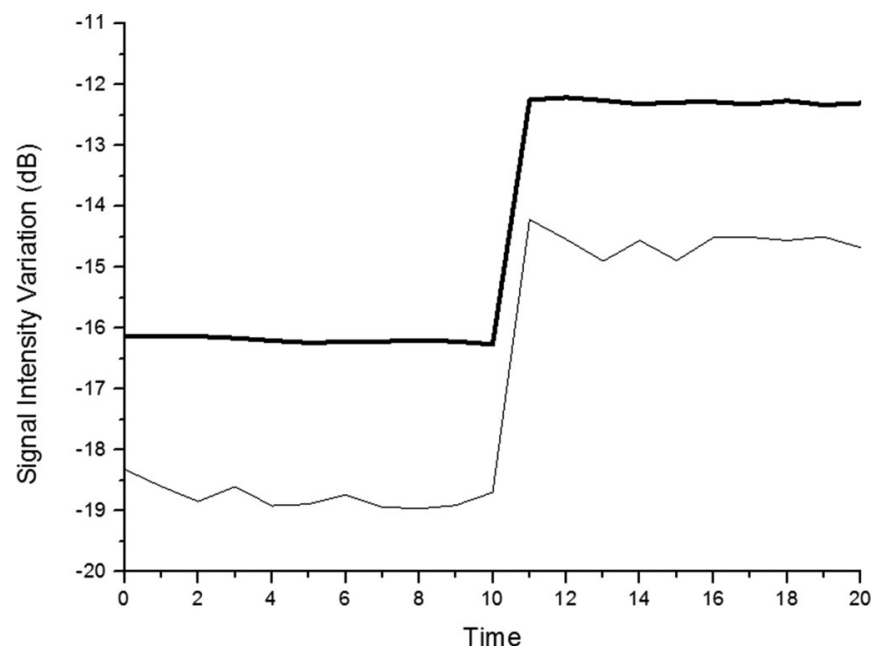

Fig. 7. Step procedure to obtain the resolution for the parallel (line) and series (bold line) interrogation systems.
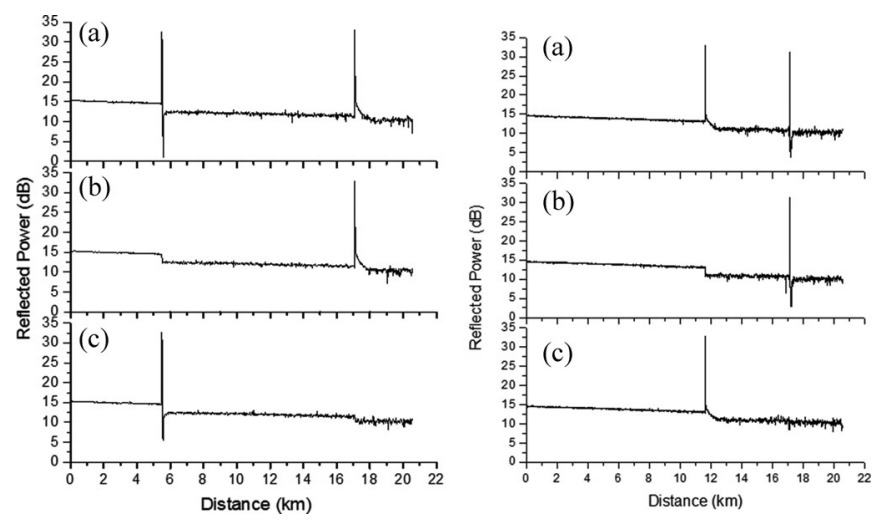

Fig. 8. OTDR traces for series (left) and parallel (right) configurations with (a) two multiplexed sensors, (b) in the absence of the first sensor and FLM and (c) in the absence of the second sensor and FLM.

intensity variation, respectively, as shown in Fig. 7. Considering these values and the average $r m s$ noise amplitudes before and after the step change, it turns out a displacement resolution of 0.027 and $0.093 \mathrm{~mm}$, respectively.

\section{MultipleXing AND SENSING}

Fig. 8(a) presents the OTDR trace of two multiplexed sensors in the series (left) and parallel (right) configurations. Crosstalk between the two sensors in both configurations was analyzed. Fig. 8(b) shows the OTDR trace when the first sensor followed by a FLM is absent. The dynamic range of the second FLM reflection peak is still the same in both configurations.

Finally, Fig. 8(c) illustrates the OTDR trace when the second sensor $(\sim 17 \mathrm{~km})$ followed by a FLM is not present. The dynamic range of the first reflection peak is about the same as shown in Fig. 8(a), also for both configurations. Therefore, crosstalk is not an issue in both configurations. The signal observed between the sensors is kept constant along the fiber. Using this type of OTDR, the sample interval is $2 \mathrm{~m}$ what corresponds to the minimum distance between the two FLMs.

\section{CONCLUSION}

In this paper, it was proposed two interrogation and multiplexing systems based on OTDR for FLM intensity sensors. The sensing zones are located before the FLM setup. This configuration based on FLM allows using any type of intensity sensors. In this work a fiber taper was used as a displacement sensor. The FLM structure when is monitored through the OTDR generates an intense peak reflection which is observed in the OTDR display. The schemes were characterized in order to obtain the maximum dynamic range of the reflection peak. It was achieved a maximum value of $17 \mathrm{~dB}$ when input signal attenuation of around $10 \mathrm{~dB}$ is used. Pulse width of $\sim 100 \mathrm{~ns}$ enabled to attain an even better dynamic range of $\sim 18 \mathrm{~dB}$. Both configurations demonstrated similar and good linearity in a specific region of the dynamic range with a $-15.3 \mathrm{~dB} / \mathrm{mm}$ slope and a $0.027 \mathrm{~mm}$ resolution for the series configuration and $0.093 \mathrm{~mm}$ for the parallel one. The series configuration presents three times more noise comparing to the parallel configuration. This result was expected due to the intense reflected signal, which suffers further reflection back and forth. Furthermore, the parallel configuration presents a $2.5 \mathrm{~dB}$ increase in the OTDR reflection peak power. Therefore, the parallel configuration may be considered the best one.

Finally it was demonstrated that the two configurations can be used for remote sensing. The choice between both configurations will always depend on the number of sensors that we want to interrogate and the distance between them. However, a hybrid configuration is also one solution for a low number of sensors. The proposed interrogation systems showed to be feasible alternatives techniques for sensor multiplexing and remote sensing when compared with other conventional interrogation systems.

\section{REFERENCES}

[1] B. Glisic and D. Inaudi, Fibre Optic Methods for Structural Health Monitoring. West Sussex, U.K.: Wiley, 2007, pp. 27-28.

[2] L. C. G. Valente, A. M. B. Braga, A. S. Ribeiro, R. D. Regazzi, W. Ecke, C. Chojetzki, and R. Willsch, "Combined time and wavelength multiplexing technique of optical fiber grating sensor arrays using commercial OTDR equipment," IEEE Sensors J., vol. 3, no. 1, pp. 31-35, Feb. 2003.

[3] X. Gong, D. Hua, P. Zhang, L. Hu, and Y. Wang, "Alternate dual pulses technique for fiber Bragg grating ultra-multi-point strain measurement," presented at the SPIE 8th Int. Symp. Precision Eng. Meas. Instrum., Chengdu, China, 2013.

[4] O. Frazão, R. Falate, J. M. Baptista, J. L. Fabris, and J. L. Santos, "Optical bend sensor based on a long-period fiber grating monitored by an optical time-domain reflectometer," Opt. Eng., vol. 44, no. 11, pp. 110502-1-110502-3, Nov. 2005.

[5] E. Cibula and D. Donlagic, "In-line short cavity Fabry-Perot strain sensor for quasi distributed measurement utilizing standard OTDR," Opt. Exp., vol. 14, no. 15, pp. 8719-8730, Aug. 2007.

[6] P. Xu, F. Pang, N. Chen, Z. Chen, and T. Wang, "Fabry-Perot temperature sensor for quasi-distributed measurement utilizing OTDR," in Proc. Asia Pacific Opt. Fiber Sens., 2008, pp. 1-4.

[7] I.-B. Kwon, C.-Y. Kim, D.-C. Seo, and H.-C. Hwang, "Multiplexed fiber optic OTDR sensors for monitoring of soil sliding," presented at the 23rd IMEKO World Congress, Rio de Janeiro, Brazil, 2006.

[8] M. Bravo, M. F. Vallejo, and M. López-Amo, "Hybrid OTDR-Fiber laser system for remote sensor multiplexing," IEEE Sensors J., vol. 12, no. 1, pp. 174-178, Jan. 2012.

[9] M. T. M. R. Giraldi, C. S. Fernandes, M. S. Ferreira, M. J. de Sousa, P. Jorge, J. C. W. A. Costa, J. L. Santos, and O. Frazão, "Interrogation 
and multiplexing system for fiber loop mirror coupled intensity sensors using OTDR," Microw. Opt. Technol. Lett., vol. 56, no. 12, pp. 2860-2864, Dec. 2014

[10] J. Yuan, C. Zhao, M. Ye, J. Kang, Z. Zhang, and S. Jin, "A Fresnel reflection-based optical fiber sensor system for remote refractive index measurement using an OTDR," Photon. Sens., vol. 4, no. 1, pp. 48-52, Jan. 2014.

[11] N. J. M. Satar, N. S. A. Rahim, and M. K. AbdRahman, "Displacement sensor for structural monitoring using optical time domain reflectometer," in Proc. AIP 1150, 2009, pp. 223-228.

[12] A. M. Hatta, H. E. Permana, H. Setijono, A. Kusumawardhani, and Sekartedjo, "Strain measurement based on SMS fiber structure sensor and OTDR," Microw. Opt. Technol. Lett., vol. 55, no. 11, pp. 2576-2578, Nov. 2013.

[13] A. M. Hatta, K. Indriawati, T. Bestariyan, T. Humada, and Sekartedjo, "SMS fiber structure for temperature measurement using an OTDR," Photon. Sens., vol. 3, no. 3, pp. 262-266, Mar. 2013.

[14] G. P. Agrawal, "Multichannel systems," in Fiber-Optic Communication Systems, 3rd ed. New York, NY, USA: Wiley, 2002, pp. 377-378.

[15] M. Bravo, J. M. Baptista, J. L. Santos, M. López-Amo, and O. Frazão, "Ultralong $250 \mathrm{~km}$ remote sensor system based on a fiber loop mirror interrogated by an optical time-domain reflectometer," Opt. Exp., vol. 36, no. 20, pp. 4059-4061, 2011.

[16] E. Delarosa, L. A. Zenteno, A. N. Starodumov, and D. Monzon, "Allfiber absolute temperature sensor using an unbalanced high-birefringence Sagnac loop," Opt. Lett., vol. 22, no. 7, pp. 481-483, Apr. 1997.

[17] A. N. Starodumov, L. A. Zenteno, D. Monzon, and E. Delarosa, "Fiber Sagnac interferometer temperature sensor," Appl. Phys. Lett. vol. 70, no. 1, pp. 19-21, Jan. 1997.

[18] M. Campbell, G. Zheng, P. A. Wallace, and A. S. Holmessmith, "A distributed frequency modulation continuous wave fiber stress sensor based on a birefringent Sagnac ring configuration," Opt. Rev., vol. 4, no. 1A, pp. 114-116, Jan./Feb. 1997.

[19] D. H. Kim and J. U. Kang, "Sagnac loop interferometer based on polarization maintaining photonic crystal fiber with reduced temperature sensitivity," Opt. Exp., vol. 12, no. 19, pp. 4490-4495, Sep. 2004.

[20] Y. Liu, B. Liu, X. H. Feng, W. G. Zhang, G. Zhou, S. Z. Yuan, G. Y. Kai and X. Y. Dong, "High-birefringence fiber loop mirrors and their applications as sensors," Appl. Opt., vol. 44, no. 12, pp. 2382-2390, Apr. 2005.

Maria Thereza Miranda Rocco Giraldi received the Graduate degree in telecommunications engineering from Federal Fluminense University, Niteroi, Brazil, in 1986, and the master's and Ph.D. degree in electrical engineering from Pontifical Catholic University, Rio de Janeiro, Brazil, and Universidade Estadual de Campinas, Campinas, Brazil, in 1989 and 1995, respectively. She is an Associate Professor at the Military Institute of Engineering from the Brazilian Army. She is a Researcher of the Brazilian National Council of Scientific and Technological Development. Her research interests include optical communication and sensors, more specifically with optical devices and systems, WDM systems, free-space optics communications, optical amplifiers, and fiber sensors. She is the Editor-in-Chief of the Journal of Microwaves, Optoelectronics and Electromagnetic Applications, a journal published by the Brazilian Microwave and Optoelectronics Society and the Brazilian Electromagnetics Society.

Cindy Stella Fernandes received the graduate degree in telecommunications engineering from the Institute for Higher Education of Amazon, Belém, Brazil, in 2005, and themaster's degree in mechanical engineering from the Federa University of Pará, Belém, in 2011. She is currently working toward the Ph.D. degree in electrical engineering at the Applied Electromagnetism Laboratory, Federal University of Pará. She has been working with optical communication and sensors, more specifically with optical devices, systems, and fiber sensors.

Marta S. Ferreira received the graduate degree and the M.Sc. degree in physical engineering in 2009 from the University of Aveiro, Aveiro, Portugal. She is currently working toward the $\mathrm{Ph} . \mathrm{D}$. degree at the Optoelectronics and Electronic Systems Unit, Institute for Systems and Computer Engineering of Porto, Porto, Portugal, and the Faculty of Sciences of the University of Porto. She has published several papers in international journals and conferences. Her scientific research interests include the postprocessing of optical fibers, fiber sensing, and fiber lasers. She is a Student Member of SPIE and OSA.
Marco José de Sousa received the M.Sc. and Ph.D. degrees in electrical engineering from the Federal University of Pará (UFPA), Belém, Brazil, in 2003 and 2008, respectively. He received the title of Electrical Engineer by UFPA, in 2002. He is a Member of the Applied Electromagnetism Laboratory of UFPA since 1999. He joined UFPA as an Assistant Professor in 2009, where he is currently working in the area of sensors and embedded systems. His research interests include optimization, optical communications, optical sensors systems, optoelectronic devices, and embedded systems.

Pedro A. S. Jorge received the graduate degree in applied physics (optics and lasers) from the University of Minho, Minho, Portugal, and the M.Sc. degree in optoelectronics and lasers from the Physics Department of the University of Porto, Porto, Portugal, in 1996 and 2000, respectively. In 2006, he concluded his $\mathrm{Ph} . \mathrm{D}$. program at the University of Porto in collaboration with the Department of Physics and Optical Sciences at the University of Charlotte, NC, USA. He is currently a Senior Researcher at the Institute for Systems and Computer Engineering of Porto, Porto, where he leads the Biochemical Sensors team exploring the potential of optical fiber and integrated optics technologies in environmental and medical applications coordinating several projects in the area. He has more than 150 publications in the fields of sensors in national and international conferences and peer-reviewed journals, is an author of two book chapters and also holds one patent

João Crisóstomo Weyl Albuquerque Costa received the M.Sc. degree in electrical engineering from the Pontifical Catholic University of Rio de Janeiro, Rio de Janeiro, Brazil, in 1989, and the Ph.D. degree from the State University of Campinas, Campinas, Brazil, in 1994. He has been with the Federal University of Pará (UFPA), Belém, Brazil, since 1994, where he is the Head of the Applied Electromagnetism Laboratory and affiliated to the Computer Science and Electrical Engineering Postgraduation programs. He was the Head of the Graduate Program in electrical engineering from 2004 to 2005 and the Research Director of UFPA from 1999 to 2001 and from 2005 to 2006. From 2007 to 2010, he was the Deputy Secretary for Development, Science and Technology, coordinating the program of integration of the Pará State using optical networking and broadband access via wireless networks. His research interests include the areas of wireless, optical and access networks, and applied electromagnetism. $\mathrm{He}$ is the President of the Brazilian Microwave and Optoelectronics Society from 2012 to 2014, a Member of the Brazilian Society of Telecommunications, and a Researcher of the Brazilian National Council of Scientific and Technological Development. He is the Vice President of the Federal University of South and Southeast Pará since August 2013.

José Luís Campos Oliveira Santos received the graduate degree in Physics (optics and electronics) and the Ph.D. degree and Habilitation from the University of Porto, Porto, Portugal, in 1983, 1993, and 2008, respectively. His main research interests include optical fiber sensing and optical fiber technology. He holds the position of Full Professor of Physics and Astronomy Department of University of Porto and is a Researcher of INESC Porto Optoelectronics and Electronic Systems Unit. He is a Member of OSA, SPIE, and Planetary Society.

Orlando Frazão received the graduate degree in physical engineering from the University of Aveiro, Aveiro, Portugal . and the Ph. D. degree in physics from the University of Porto, Porto, Portugal. From 1997 to 1998, he was with the Institute of Telecommunications, Aveiro. He is currently an Invited Assistant Professor with the Department of Physics of Faculty of Science, University of Porto, and he is also a Senior Researcher with Optoelectronics and Electronic Systems Unit, Institute for Systems and Computer Engineering of Porto, Porto. His present research interests include optical fiber sensors and optical communications. $\mathrm{He}$ has more than 300 papers in international journals and conferences. $\mathrm{He}$ participated as organized committee of several International conferences. $\mathrm{He}$ received three scientific awards. He is a Senior Member of the SPIE and OSA. 